\title{
Corps de tyran, corps de roi : la dialectique de body et de cor(p)se dans le théâtre shakespearien
}

\section{Raymond Gardette}

\section{(2) OpenEdition}

Journals

\section{Édition électronique}

URL : http://journals.openedition.org/shakespeare/1191

DOI : 10.4000/shakespeare.1191

ISSN : 2271-6424

Éditeur

Société Française Shakespeare

\section{Édition imprimée}

Date de publication : 1 novembre 1991

Pagination : 81-99

Référence électronique

Raymond Gardette, "Corps de tyran, corps de roi : la dialectique de body et de cor(p)se dans le théâtre shakespearien », Actes des congrès de la Société française Shakespeare [En ligne], 9 | 1991, mis en ligne le 01 janvier 2007, consulté le 06 mai 2019. URL : http://journals.openedition.org/shakespeare/1191 ; DOI : 10.4000/shakespeare.1191 


\section{SHAKESPEARE \\ ET LE CORPS A LA RENAISSANCE}

Société Française Shakespeare

Actes du Congrès 1990

sous la direction

de

M.T. JONES-DAVIES

PARIS

LES BELLES LETTRES

1991 
Tous droits de traduction, de reproduction et d'adaptation réservés pour tous les pays.

(C) 1991 Société d'édition Les Belles Lettres, 95 bd Raspail 75006 Paris

ISBN $2.25169117-\mathrm{X}$ 


\section{Corps de tyran, corps de roi : la dialectique de body et de cor(p)se dans le théâtre shakespearien}

Les jurisconsultes du Moyen-Age et les historiographes et monarchomarques du XVI $\mathrm{X}^{e}$ siècle ont vu dans la persécution des âmes la forme la plus perverse du pouvoir tyrannique, assimilé, depuis la plus haute Antiquité, à l'usage illimité d'une responsabilité délimitée - tyrannie, imperium, royauté, magistrature soit sans la garantie des restrictions établies par la Loi' ${ }^{1}$. Le refus de respecter le contrat avec le corpus politicum ou le corpus mysticum - d'usage équivalent dans la société médiévale ${ }^{2}$-, véritable atteinte aux droits de l'Etat et du peuple, est une injure à l'organisation divine du monde. Selon Thomas Smith, la tyrannie s'exerce sur trois pôles, selon qu'il s'agit de la manière d'arriver au pouvoir par la force, d'introduire des lois sans respecter la volonté du peuple, ou de porter atteinte au corps politique de façon à le confondre avec sa propre personne ou sa famille - dans chaque cas une attitude sacrilège ${ }^{3}$. Dans le contexte des guerres religieuses du $\mathrm{XVI}^{e}$ siècle européen, la question du tyrannicide s'est posée en termes ontologiques. Sous l'influence de l'emblématisation populaire de la descente aux enfers - danse des morts ou représentation du Jugement Dernier - la croyance que le cadavre ou le squelette d'un meurtrier est toujours habité des forces démoniaques qui le possédaient, que l'assassin même mort demeure un assassin, conduit à lui faire subir un martyre rituel et purificatoire ${ }^{4}$, inverse exact du rite accompagnant le "corps royal éviscéré, embaumé, redoublé d'une effigie" s'il agit d'un cas de lèse-majestés. La superstition concernant les prodiges ou événements surnaturels accompagnant tout régicide ou tyrannicide repose sur la croyance que le sang versé attire le sang, que le meurtrier du tyran est susceptible d'être accusé de tyrannie. Cela explique l'inversion des rites funéraires par laquelle on tente d'annihiler les forces maléfiques, par le démembrement, voire l'avalage d'un morceau de la chair honnie ${ }^{6}$. Il 
y a là un cannibalisme rituel que l'on peut mettre sur le même plan, si l'on en croit Montaigne, que le cannibalisme politique et religieux qui s'en prend à l'hérétique:

Je pense qu'il y a plus de barbarie à manger un homme vivant qu'à le manger mort, à deschirer, par tourments et par geénes, un corps encore plein de sentiment, le faire rostir par le menu, le faire mordre et meurtrir aux chiens et aux pourceaux (comme nous l'avons, non seulement leu, mais veu de fresche memoire, non entre des ennemis anciens, mais entre des voisins et concitoyens, et, qui pis est, sous pretexte de pieté et de religion), que de le rostir et manger après qu'il est trespassé.?

L'attrait pour la découverte physique du corps, de son fonctionnement, de ses mystères, de ses maladies, de ses merveilles ou vices cachés ${ }^{8}$ se retrouve dans l'attitude humaniste qui relie l'âme au corps de façon indissoluble. La préoccupation qui consiste à préférer l'âme au corps a une traduction sémantique, dans quelques pièces de Shakespeare, sous la forme d'une dichotomie entre les vocables body et cor $(p)$ se ou - dans la graphie du XIVe siècle corpes/corpse : d'un côté le corps propre, vital, biologique, naturel, de l'autre le corps représenté, dépouille éviscérée, effigie - comme dans les cérémonies funéraires royales - ou encore fantôme. Dans Roméo et Juliette, où la haine détruit les corps, la parade inventée par frère Laurence consiste à retirer un moment l'apparence de la vie au corps de l'héroïne, à la transformer en effigie reposant dans un tombeau : «Poore living Coarse, clos'd in a dead mans Tombe» ${ }^{9}$. Juliet est alors désignée du même qualificatif que le corps de Tybalt qui, lui, est mort et saigne en présence de son meurtrier, selon une croyance populaire que Shakespeare exploite par ailleurs ${ }^{10}$. Ainsi le texte shakespearien glose-t-il l'échange tragique entre Eros et Thanatos. C'est néanmoins dans les pièces romaines et historiques que le jeu sémantique sur les corps est le plus apparent. Dans Henri IV - première partie, la victoire de Harry Lancaster sur Harry Hotspur est décrite sous la forme d'un passage de body à $\operatorname{cor}(p)$ se. La gémellité des deux princes permet d'interpréter l'événement miraculeux comme la domination du corps mystique sur le corps naturel du futur roi. Le rite sauvage auquel se livrent les Galloises sur les "dead corses" des soldats de Mortimer, qu'elles démembrent ou émasculent, est un tyrannicide symbolique. Elles interdisent toute succession légitime établie par la force au bénéfice de la maison de Lancastre. La mort d'Hotspur, 
où Henri IV - Bolingbroke retrouve l'image de ce qu'il fut à Ravenspurgh lors de la déposition de Richard II, traduit l'impossibilité de la légitimité d'un fils - à la Bolinbroke ${ }^{11}$. De façon plus systématique, les modalités de la prise du pouvoir, les nécessités et les aléas du tyrannicide, les modes de la vengeance politique font l'objet de modulations sémantiques sur la corporéité. Il en est ainsi de Coriolan, Jules César, Hamlet, Richard III.

Dans le registre de l'imaginaire politique du XVI ${ }^{\mathrm{e}}$ siècle, où s'établissent des rapports dialectiques entre physis et polis ${ }^{12}$, Coriolan donne l'exemple d'une construction dramaturgique ordonnée autour de la vaine tentative chez les patriciens, d'associer l'espacecorps physique et l'espace-corps politique. La tragédie décrit l'impossible isomorphisme entre deux corps - Coriolan couvert de blessures, garant de la protection de Rome, véritable allégorie dramatique d'une guerre de survie ${ }^{13}$, et la Cité, décrite par l'entremise du célèbre apologue du ventre et de l'estomac ${ }^{14}$ et grâce aux analogies platoniciennes ${ }^{15}$ comme un corps vivant. Le divorce s'établit entre un irréel politique - "a pretty tale", que les tribuns se montrent aussi peu enclins à utiliser qu'est avide de s'y référer Menenius Agrippa - et une construction politique morcelée. La tentative des sénateurs de distraire les citoyens affamés de la misère de leur corps biologique - «To fobbe off our disgrace with a tale», selon la formule du premier citoyen ${ }^{16}$ - se révèle grossière et inopérante. Ce refuge dans l'Imaginaire, auquel ont recours les Patriciens, se heurte à la «contre-épreuve du réel établi» ${ }^{17}$. Dans une démarche inverse, et au terme de l'itinéraire qui conduit Coriolan de Rome à Corioles, de la gloire célébrée à la mort indigne, la contre-épreuve de l'Imaginaire, à l'initiative des conspirateurs, le temps de la mise à mort, réussit à éluder l'épreuve, et les preuves, de I'Histoire établie. «If you have writ your Annales true», clame en vain Coriolan à l'adresse d'Aufidius ${ }^{18}$. Les Volsques, en définitive, s'associent aux Romains dans le refus d'établir une relation dialectique entre le corps du héros sauveur et le corps de la Cité à sauver. La défaite finale de Coriolan est dans la rupture entre les mots et les actes. La rhétorique du pouvoir, toujours susceptible d'être ressentie comme une forme de tyrannie ${ }^{19}$, est retirée au héros déchu. La conspiration, qui veut sa mort, lui interdit de se justifier auprès du peuple - «To purge himself with words», dit Aufidius $^{20}$ - et, dès lors, la question de l'attitude tyrannique du protagoniste demeure posée. Les deux seules accusations de tyrannie adressées à l'encontre de Coriolan - l'affectation de "pouvoir 
tyrannique", que le tribun Brutus entend introduire dans les chefs d'accusation contre le sauveur de Rome est, plus encore, le sentiment qu'a Coriolan d'exercer une tyrannie envers sa mère, sa femme et Rome, au point de se décrire comme un acteur oublieux de son rôle ${ }^{21}$ - apparaissent, au moment de sa mort, comme de maladroites antiphrases. A l'heure du sacrifice, le héros devenu victime n'est pas ce qu'on a dit ni ce qu'il a cru qu'il était. Aussi bien la précipitation des conspirateurs, soucieux d'écarter les réflexes de justice des Seigneurs de Corioles, s'explique par la crainte qu'ils ont d'une possible relaton dialectique entre le corps communautaire - "th'body of the weal", selon la formule du tribun Brutus - et le corps héroïque du héros. C'est ce qu'explique, avec cynisme, le troisième conspirateur: «... when he lies along / After your way. His Tale pronounc'd, shall bury / His Reasons with his body»22. Il est ironique que Coriolan emporte dans la tombe la fable du corps politique dont il n'est pas le théoricien. A cet égard, l'exécution sacrificielle de ce bouc émissaire est une tragédie d'ordre personnel et non politique. Elle n'entraîne aucune purification de la polis. Le cérémonial funèbre, auquel se rangent avec des réserves Aufidius et le deuxième Lord, est, pour cette raison même, ambigu. La rhétorique funèbre est contredite par le cérémonial funéraire. Le Verbe, certes, transfigure le héros, dont le body, devenu corpse, est porté en procession, comme dans les cérémonies d'enterrements royaux, où la victoire sur la mort est signifiée par l'effigie royale ${ }^{23}$ :

I Lord. Bear from hence his body, And mourne you for him. Let him be regarded

As the most noble coarse, that ever Herald

Did follow to his Ume. ${ }^{24}$

Sur le plan de la représentation, néanmoins, l'emprunt au rituel funéraire des rois n'est qu'apparent. Coriolan est porté par quatre soldats, dont Aufidius, symbolique thêâtrale qui rappelle le privilège accordé aux "quatre présidents du Parlement" lors d'un enterrement royal, de porter les cordons du poêle pour signifier qu'ils sont "partie du corps du roi"25. Mais Aufidius, instigateur de l'assassinat politique, apparaît comme un usurpateur et non comme un juge éminent. La duplicité qui s'est établie à Corioles se cache vainement derrière l'imitation du rituel funéraire par lequel se perpétue la doctrine, établie par le droit public français, que "le Roi ne meurt jamais" 26 . A Corioles, il ne se produit aucune transmission 
de pouvoir, à la façon dont Hamlet, par exemple, désigne son successeur avant d'être symboliquement porté par quatre capitaines, comme le Roi qu'il eût mérité d'être ${ }^{27}$. Le cérémonial funéraire qui accompagne Coriolan dans la mort, s'il dissocie le corps naturel de l'effigie du héros, n'entre dans aucune problématique de succession. Dans le Coriolan de Shakespeare, un héros accusé, semble-t-il à tort, de tyrannie, meurt pour rien.

Dans la mise en scène shakespearienne de la mort de César ${ }^{28}$, un jeu dialectique s'organise autour de la synergie du "corps représenté" 29 - en l'occurrence celui de César - et de la rhétorique corporelle à laquelle se livrent les conjurés. Avant son exécution sacrificielle, le roi que Rome vient d'élire ne cache pas sa surdité, ce qui amoindrit l'effet de la mise en cause de sa faiblesse physique par Cassius. En fait, les accusations de tyrannie, contrebalancées par la romanitas dont César fait montre, ne sont guère justifiées que par implication, voire calomnie. Cassius décrit une crise d'épilepsie dont aurait été victime César, ce que n'atteste pas l'Histoi$\mathrm{re}^{30}$, en faisant naître à l'esprit de Brutus l'image visuelle d'un "corps absent" 31 . Epicurien qu'envahit la mélancolie, Cassius donne limpression de porter, sur des épaules qu'il dit être plus fortes que celles de César, le poids des fautes qui, selon les conceptions antiques de la tyrannie, hantent le tyran et le détruisent de l'intérieur ${ }^{32}$. Son apologie du suicide comme moyen d'échapper à la tyrannie ${ }^{33}$, à la manière sénéquéenne, le conduit à adopter une fausse attitude stoïcienne ${ }^{34}$. Par contre, la progression de l'idée de suicide est exemplaire chez Brutus, amenée à prendre sa vie parce que les conditions politiques ne permettent plus l'exercice de la Vertu. Il se tue parce que la tyrannie continue malgré le tyrannicide. Il découvre que César est plus présent mort que vif. Et l'illusion que dénoncent les suicides successifs de Portia, Cassius, Titinius et Brutus, c'est la découverte de l'impossible dissociation du corps et de l'esprit de César. «O that we then could come by Caesars Spirit, / And not dismember Caesar !» : l'esprit de César survit à sa disparition physique, contrairement aux espoirs de ses assassins $^{35}$. Dans la perspective providentielle inspirée de Plutarque, les conjurés sont saisis de crainte face à des prodiges où se lit la colère du ciel contre les puissants de ce monde ${ }^{36}$. Calphurnia, en pythonisse, à la veille du tyrannicide, puis Cassius, parjure de l'Epicurisme, à la veille de Philippes, annoncent tous les deux que l'heure de la mort est fixee par la Providence ${ }^{37}$. Dans ce contexte, 
le corps en représentation - celui de Cassius, d'abord joué contre celui de César, avant d'être transformé en cadavre anonyme sur le champ de bataille et, de façon antinomique, celui de César, que Brutus, à la demande d'Antony, accepte d'offrir à la vue du peuple, que le premier plébéien entend brûler "dans le lieu sacré" et qui s'imprime dans la mémoire de Brutus $^{38}$, ce corps dans sa matérialité biologique apparaît comme le référent dramatique d'une manifestation d'ordre spirituel. L'ambiguïté de la démarche des tyrannicides est mise à jour grâce à la projection dans l'esprit des citoyens romains de la survie de l'esprit de César au-delà de la mort. C'est ce qu'obtient Antony, grâce à la célébration funèbre qu'il organise autour de la dépouille du Roi assassiné et qui confère à cette dernière une valeur sacramentelle.. Il est ironique que Brutus emprunte à Antony la dénomination $\operatorname{cor}(p) s e^{39}$, qui suggère, précisément, la permanence de la royauté, une "métaphysis politique" plutôt que "la physis pure du Roi"40 :

Do grace to Caesars Corpes, and grace his Speech

Tending to Caesars Glories, which Marke Antony

(By our permission) is allow'd to make. ${ }^{41}$

Antony a beau jeu d'exhorter ses concitoyens, après qu'il a pris soin d'associer le Bien fait par César à sa dépouille terrestre, au contraire du Mal qui survit à la disparition des méchants ${ }^{42}$, à faire cercle autour du "corpes" de César :

Then make a Ring about the Corpes of Caesar And let me chew you him that made the Will. ${ }^{43}$

La symbolique du cercle ou de la couronne, corrélat de la personne du Roi et image de l'unité du corps politique, est utilisée sciemment, dans le cadre d'une gestuelle emblématique. Il s'agit d'empêcher la privation du droit à la couronne du peuple tout entier, la disherison reprochée à Richard II et à d'autres ${ }^{44}$. Antony fait la démonstration que nul ne saurait s'approprier à des fins personnelles la couronne royale, signe d'un pouvoir qui appartient au peuple. Là serait la vraie tyrannie. La réponse au dilemme que pose Antony est dans le jugement divin qui finit par s'exercer contre les tyrannicides; Sur la scène shakespearienne, le mauvais "génie" de Brutus est représenté par le fantôme de César, figuration dramatique non de la vengeance mais de "l'âme tyrannisée" 45 de Brutus, perturbée quant à la survie de la Justice dans l'après-César. Le lien, qui s'établit entre le mort et le vif et que représente la métaphore 
dramatique, signifie que le tyrannicide Brutus est devenu, à son tour, le réceptacle de la tyrannie. La seule issue possible, pour le Stoïque, c'est le suicide. C'est dans cette perspective que l'on peut dire de l'apparition de César qu'il est un fantôme de justice distributive.

Dans la pensée stoïcienne, augures et prodiges sont la révélation et non une cause de bouleversements naturels, sociaux, politiques qu'ils annoncent ${ }^{46}$. De la même façon pourrait-on dire, les fantômes qui apparaissent sur les scènes des XVI ${ }^{e}$ et XVII ${ }^{e}$ siècles constituent non la raison mais le signe révélateur du Surnaturel. Ils ne sont que des éléments d'une opsis, où se lit une interrogation métaphysique. L'influence de la doctrine chrétienne, plus que celle de la pensée antique, s'y retrouve, au-delà des emprunts du théâtre de la Renaissance aux oripeaux du thêâtre sénéquéen.

Les manifestations surnaturelles, dans le thêâtre élisabéthain et jacobéen relèvent, semble-t-il, d'un syncrétisme où les schèmes de l'Antiquité s'enrichissent des doctrines physiologiques du Moyen-Age et de la Renaissance, où l'Homme est décrit dans un va-et-vient entre le monde corporel, matériel, visible et le monde immanent, immatériel, invisible ${ }^{47}$. Les pneumatologues de la Renaissance s'interrogent sur trois sortes d'apparitions :

1 - l'ombre -umbra-ou spectre d'un défunt de retour sur terre, forme d'apparition que conteste la pensée protestante;

2 - la vision - visum, que les Anciens distinguent de la visio, lieu des prophéties divines - apparence de caractère illusoire, lieu privilégié des apparitions diaboliques selon l'onirologie chrétienne;

3 - l'image - imago - représentation mentale, reflet en miroir du corps et de l'âme, et donc une fabrication de l'esprit humain ${ }^{48}$. A l'époque de Shakespeare, cette image-représentation se relie à un désordre physiologique, très souvent la mélancolie ${ }^{49}$. Dans le contexte de cet infraconscient culturel, Hamlet le mélancolique s'interroge sur la signification vraie de l'apparition fantomatique de son père rejeté par son sépulcre, en habit complet de guerrier. La face signifiante du fantôme renvoie, par un effet de miroir, l'image de ce qu'il fut sur terre et aussi de ce que sera son assassin. Il est le signe que ce dernier n'est qu'un mort en sursis, qu'il sera ce que lui, fantôme, est devenu. Il est une image de la fin inexorable de chaque être humain. L'influence des traditions figuratives médiévales, où la mort en forme de squelette vient signifier l'inévitable 
échéance, s'y retrouve. Entre autres figurations, la danse macabre - où chaque squelette en personnage, face à son corrélat humain et revêtu des signes sociaux distinctifs de ce dernier, lui signifie qu'il est un mort en sursis - est présente en filigrane dans la confrontation scénique entre un fantôme et son interlocuteur privilégié. Dans Hamlet, la dialectique qui s'établit entre les deux corps, le vif et le mort, est d'ordre sémantique autant que dramatique. Le fantôme relève de l'ambiguïté qu'établit Claudius entre le signifiant et le signifié lorsque, reprochant à Hamlet, qui pleure la mort de son père, de ne pas respecter la facture divine du mon$\mathrm{de}^{50}$, il associe le coarse de la victime du premier fratricide au roi Hamlet qui vient de mourir - «From the first Coarse till he that dyed to day ${ }^{51}$. La résurgence dans la mémoire du meurtrier usurpateur du crime archétypal de Cain est un référent rhétorique au signe thêâtral qu'est le fantôme. Hamlet, quant à lui, est conduit à voir dans le fantôme de son père une représentation pneumatologique dont la signification commence par lui échapper :

... what may this meane

That thou dead coarse again in compleat steele

Revisits thus the glimpses of the Moone

Making Night hidious? And we fooles of Nature,

So horridly to shake our disposition

With thoughts beyond the reaches of our soul,

Say, why is this? Wherefore? What should we doe? ${ }^{52}$

La confrontation du fils et de son père défunt, par delà la frontière qui sépare les vivants et les morts, à l'insu des autres protagonistes, peut se lire comme l'emblème dramatique d'une transmission de pouvoir du mort au vif. A l'instar d'une figuration de danse macabre, le fantôme, sinon le squelette, en personnage, signifie que la majesté royale, loin de se réduire aux insignes visibles du pouvoir, est, au contraire, distincte de toute corporalité. Dans une macabre logique, Hamlet se voit confier la tâche de s'intercaler

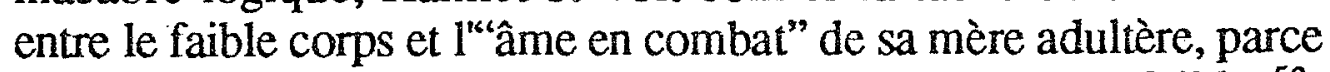
que l'imagination est la plus à l'œuvre dans les corps faibles ${ }^{53}$. Celle-ci s'étonne de le voir tenir des discours à "l'air incorporel" et refuse de découvrir dans la vision du roi Hamlet, que veut lui imposer son fils, autre chose qu'une mélancolique vision : «Ces créatures sans corps / Le délire excelle à les forger» ${ }^{54}$, risque-t-elle. Mais que le corps fantasmé du vrai roi entre en conflit avec le corps manifeste du roi usurpateur, l'un étant l'image usurpée de 
l'autre ${ }^{55}$, comme s'acharne à le démontrer Hamlet, et le fantôme, ou fantasme, envahit l'esprit de Gertrude. La dissociation du corps et de l'âme hante Hamlet à un point tel qu'il en attribue les effets au "corps du Temps" dont il demande aux comédiens de révéler l'effigie, derrière les apparences usurpatrices. Devant la monstruosité de l'adultère de sa mère, il apparaît encore au jeune prince que tout contrat de mariage est le corrélat légal d'une dissociation de l'esprit et du corps ${ }^{56}$. Dans Hamlet, le corps de l'inceste ne fait qu'un avec celui de l'usurpation. Son excision est la seule condition de survie du corps politique. Le corps biologique de chaque intervenant dans la tragédie de succession royale et familiale est réduit, par là-même, à une simple enveloppe charnelle. Le commentaire désabusé du fossoyeur sur «your whoreson dead body» 57 , s'applique à chacune des écorces corporelles prise dans sa dimension temporelle. Côtoyer Claudius c'est signer son acte de mort. La seule mention de body - au sens de présence physiologique ou de corps naturel - de chaque protagoniste royal est l'evocation de sa disparition physique - qu'il s'agisse de Polonius, encombrant objet scénique après qu'il a été tué par erreur, de Rosencrantz-etGuildenstern, dont la mise à mort ne laisse aucune trace visible, de Gertrude et de Laërte, empoisonnés par le poison prévu pour Hamlet, de Claudius qu'Hamlet entrâ̂ne à s'unir à sa mère dans la mort, d'Hamlet lui-même prêt à échanger son corps mortel contre une forme fantomatique, au risque de subir les affres du poison, ce qui finit, tragiquement, par se produire ${ }^{58}$. Le moment de la révélation est celui de la mort. Dans un tel contexte, la dévaluation de body par rapport à cor(p)se est le référent sémantique du lent travail de l'empoisonnement des esprits, que traduit la métaphore dramatique de la décomposition de la chair sous l'effet du poison. Le jeu sémantique traduit la scission entre "l'immondice de la chair" et "le salut de l'homme"59. La seule protagoniste à échapper à cette dissociation, c'est Ophélie, privée de sépulture et de rites funéraires chrétiens, mais élevée paradoxalement à la dignité de cor(p)se. Le fossoyeur utilise, dans un même souffle, les mots cor(p)se et bo$d y$, pour décrire les restes humains vérolés qui ne résistent pas au pourrissement de la terre. Hamlet, à sa suite, attribue à Ophélie, dont il ne reconnaît pas la dépouille, le qualificatif noble $\operatorname{cor}(p) s e^{60}$. La fosse que dégage le fossoyeur est commune, puisqu'elle abrite entre autres, le crâne de Yorick. Elle est donc symbolique - comme dans les représentations iconographiques de l'ars moriendi où têtes et corps apparaissent depuis des tombes creusées 
à même la terre pour signifier la résurrection des corps ${ }^{61}-$ de la bicorporalité des cadavres. L'exemple du fantôme prouve que l'écorce corporelle n'est qu'apparence et qu'une liaison des âmes peut s'effectuer par l'entremise de la disparition du corps vivant. Hamlet est préoccupé par la bi-corporéité, une fois qu'il s'est convaincu de la culpabilité de son beau-père, au point de voir dans le corps naturel d'un roi ou d'un héros le calcul de ce qu'il sera après la mort, la simple ombre portée d'un corps de gueux. A Rosencrantz qui déclare que l'ambition n'est que l'ombre d'une ombre, le prince héritier de Danemark réplique, sous le couvert des paradoxes qu'autorise la folie simulée :

Then are our Beggers bodies; and our Monarchs and outstretcht heroes the Beggers Shadowes ${ }^{62}$.

Dans Hamlet, une danse macabre s'organise, au cours de laquelle le fantôme joue le rôle de la mort. Le jeune prince, son fils, en explicite, pour sa propre édification, le déroulement et il y est luimême entraîné malgré lui. La tragédie décrit la fin inévitabie des hommes, grands ou petits, où les morts innocents apparaissent comme le prix à payer pour que cesse l'usurpation politique ou charnelle, la persécution des âmes ou des corps. Le salut des âmes est à ce prix.

Selon les pamphlets sur le tyrannicide, nés des controverses et des guerres de religion dans l'Europe du XVI ${ }^{e}$ siècle, le tyran voué à la vindicte publique est celui qui rompt l'Alliance avec Dieu ou le contrat avec le peuple - les deux parjures étant indissolublement liés. Dans le premier la tyrannie est exemplaire, car le roi confond les deux juridictions, divine et royale. Sa souveraineté ne s'exerce que sur le corps et les biens de ses sujets, jamais sur leur âme, ainsi que le rappellent, entre autres pamphlets, les Vindiciae contra tyrannos:

Voila le crime de lese Majesté et de vraye tyranie, à l'occasion dequoy le Seigneur a griefvement chastié les Rois Payens mesmes. Il faut donc que les Princes qui se veulent garentir d'un si énorme cas, distinguent leur juridiction d'avec celle de Dieu, voire autant plus soigneusement que Dieu et le Prince ont leur droit tous deux sur une mesme terre, sur un mesme home, sur une mesme chose. ${ }^{63}$

Dans le deuxième cas, celui d'une rupture de fidélité envers le peuple, les pamphlétaires s'accordent à déclarer illégitime le roi qui 
contrevient à ce que Buchanan, au tournant du siècle, pour l'édification de Jacques Ir, nomme, en opposition aux théories machiavéliennes - dans un ouvrage condamné par le Privy Council d'Ecosse parce qu'il établit le principe d'une justification légale du tyrannicide - "la loi naturelle"64. L'avertissement, dans la droite ligne des formulations des jurisconsultes médiévaux quant à la prééminence de Lex sur Rex, donna lieu, de la part du souverain Stuart, à des déclarations solennelles à son Parlement pour démontrer qu'ayant reçu le sacrement de l'onction au nom de son peuple, il ne saurait accepter lidée d'un peuple au service de son souverain et non à celui de Dieu ${ }^{65}$. Néanmoins, la tentation de l'absolutisme survécut au XVI' siècle et les souverains anglais du $\mathrm{XVII}^{\mathrm{e}}$ siècle eurent du mal à associer le dogme de l'obéissance à Dieu et la notion de souveraineté établie à partir de la personnification juridique de l'ensemble du peuple. En témoigne l'hésitation de Jacques Ier à croire en la vertu thaumaturgique des rois ${ }^{66}$. $\mathrm{La}$ résistance huguenote sut utiliser l'argument religieux et le mettre au service de l'Etat, dans sa définition nouvelle de pays ou de royaume ${ }^{67}$. Les deux tétralogies shakespeariennes consacrées à la guerre des Deux Roses, écrites et jouées dans la dernière décennie du XVI ${ }^{e}$ siècle $^{68}$, constituent un témoignage de la hantise tudorienne quant à l'existence de tyrans d'exercice dans le cadre d'une vision providentielle de l'Histoire. Les chroniqueurs anglais contemporains de Shakespeare gardent le silence sur la démise du roi Richard II, mais ils s'accordent à voir dans la défaite et la mort du roi Richard III, dont le règne est le fruit de la longue suite de guerres civiles issues de la déposition d'un roi légitime, une juste punition divine. Dans la version shakespearienne, cette tyrannie d'exercice apparait, au regard des règnes Lancastriens, comme une parenthèse de l'Histoire, la nécessaire contrition d'un peuple avant l'apparition providentielle du premier des Tudors. Refus obstiné de l'ordre divin, le règne sanguinaire de Richard III se recouvre avec cynisme du manteau de la religion. Le sardonisme du tyran s'exerce à l'encontre de Dieu autant qu'à l'égard de ses ennemis temporels. Le Richard III shakespearien est, en quelque sorte, un mock-king non pas dans un contexte de fête, dont la vertu est purificatrice ${ }^{69}$, mais dans une véritable saturnale tragique, où la difformité du corps du tyran est le signe dramatique de la monstruosité du corps politique. La distorsion tyrannique va jusqu'à faire du Laid le Beau, de la Haine l'Amour et de la Mort l'équivalent de la vie. Car Richard, lointain émule de Caligula, voit dans toute créature une 
proie en puissance. Son désir de soumission des êtres à sa volonté de puissance est tel qu'il entend faire accompagner la destruciton du corps de ses victimes par le viol de leur conscience, y compris audelà de la barrière de la vie - ce qui en fait un tyran exemplaire. Cette usurpation de l'intégrité par la difformité, du divin par l'humain, trouve un corrélat sêmantique dans l'usage ambigu du qualitatif $\operatorname{cor}(p)$ se. A la manière de la Mort individualisée qui, dans une danse macabre, accompagne le contrepoint humain dont elle emprunte les signes distinctifs, le tyran entend rappeler à chacun qu'il n'est qu'un mort en puissance. Son pouvoir s'exerce même à l'encontre de la dépouille du roi qu'il vient d'assassiner. Anne Neville qui accompagne le cercueil d'Henri VI, se voit interdire par Richard de participer au rite funéraire royal. Le tyran joue sur la polysémie de cor(p)se - cadavre, effigie ou fantôme - ${ }^{70}$, alors qu'Anne tente vainement d'introduire la distinction entre le corps physique et le corps éternel du roi :

Avant thou dreadfull minister of Hell;

Thou had'st but power over his Mortall body,

His Soule thou canst not have : Therefore be gone. ${ }^{71}$

Que Richard réussisse à s'insérer entre le corps et l'âme de cette nouvelle victime dont il fait son épouse, après en avoir tué et le père et l'époux, est le signe du pouvoir démoniaque qu'il exerce. Il organise sa propre danse macabre en transformant le rituel du mariage en une marche forcée vers la mort, de même qu'il fait de l'assassinat politique un motif de religiosité. C'est avec un air de dévotion contrite qu'il réclame pour son frère Clarence, qu'il vient de faire assassiner, le droit à un cérémonial funéraire, au moment même où il apporte la nouvelle de sa mort:

Who knows not that the gentle Duke is dead?

You do him injuries to scorn his Coarse.

(They all start) ${ }^{72}$

La sanglante mascarade ne prend fin que lorsque Richmond signifie à Richard, qui n'a cessé d'emprunter le rôle de la Mort dans une façon de danse macabre dramatisée, que l'heure est venue d'échanger les rôles et de devenir, à son tour, un simple mortel appelé à devenir squelette. Le futur Henri VII, inspiré de Dieu, sait que le corps physique de Richard cache un mort en sursis et qu'il lui appartient de réduire le tyran à la loi commune: 
For me, the ransome of my bold attempt

Shall be this cold Corpes on the earth's cold face ${ }^{73}$.

La formulation, qui semble empruntée à Richard traduit, grâce à la dévaluation sémantique que Richmond impose à "corpes", la fin de la transgression tyrannique, usurpatrice de la loi divine. La thêâtralisation de ce jeu sémantique est dans l'apparition des fantômes des onze victimes de Richard ${ }^{74}$. Dans une gémination symbolique, Richard puis Richmond sont soumis à ces apparitions fantomatiques, ou fantasmatiques puisqu'elles sont reliées aux variations sémantiques du langage ${ }^{75}$. Richmond y voit les âmes des corps détruits par le tyran, ce qui réintroduit le concept des deux corps :

Me thought their Soules, whose bodies Rich murther'd Came to my tent and cried on Victory ${ }^{76}$.

Dans ce contexte, le corps physique du tyran, qui relève à la fois d'une opsis et d'une mise en discours, est le signe de l'association monstrueuse de la religion et de la tyrannie - que Richard adorne son apparence difforme des insignes de la beauté, qu'il plie son corps à la génuflexion dans une mimique d'humilité chrétienne, ou qu'il le prétende ensorcelé par l'épouse et la maîtresse d'Edouard IV ${ }^{77}$. Quant au corps uniquement parlé, il est le corps - déjà absent, dans un écart entre le signe et le signifie, distance qui préfigure l'élimination physique du monstre tyrannique. Il en est ainsi des corps décrits, de Clarence par son meurtrier, d'Henri VI ou de Buckingham au lendemain ou au jour-même de leur assassinat, des nobles tués à Bosworth, de la mère de Richard III à qui la Reine Margaret adresse sa malédiction pour avoir donné naissance à une bête sauvage qui s'en prend au fruit des entrailles de sa propre mère, ou encore de chacun des deux jeunes princes assassinés, dont la mère, veuve d'Edouard, suggère à Richard, qui veut courtiser sa fille Elisabeth, d'envoyer à cette dernière un mouchoir étanché du sang de ses frères ${ }^{78}$. Dans chacun de ces cas exemplaires, le corps vital de la victime tyrannisée est le réceptacle de la cruauté. Emblème dramatique du meurtre sacrilège, il est glosé comme la preuve de l'indissolubilité des liens entre le corps et l'âme - une constante de la pensée humaniste - et, par voie de conséquence, comme preuve de l'inimitié de Dieu à l'encontre du meurtrier de son frère. 
En définitive, il semble que le tyran, dans le texte shakespearien, soit présenté dans une relation épistémologique avec le surnaturel. Lorsqu'il est confronté à des images du corps issues de l'au-delà, dans une expérience paroxystique, il découvre les limites de son existence corporelle. Confronté à des hallucinations, condamné à la perte du sommeil - figuration classique de la mort de l'âme - il est entraîné dans une mort qui s'apparente à la condamnation éternelle. Macbeth se précipite dans l'enfer, Richard III y est conduit, Gertrude en fait la découverte de son vivant, avant d'y précéder Claudius. Le tyrannicide, en tant que meurtre, s'ancre dans une démarche ontologique. Il est une conséquence, non un départ, ce que prouvent les assassins de Coriolan et de Jules César. Il est le signe thêâtral d'une série d'apories, où les acteurs, sur la scène du monde, se heurtent à l'insondable silence du Ciel.

Raymond GARDETTE Université de Paris-Sorbonne

\section{NOTES}

1. La Tyrannie, dir. de la publ. S. Goyard-Fabre, Cahiers de philosophie politique et juridique, 6, (Centre de Publications de l'Univ. de Caen, 1984).

2. E.H. Kantorowicz, Mourir pour la Patrie et autres textes, trad. française de L. Mayali et M. Schütz (Paris, PUF, 1984): "Mystère de l'Etat. Un concept absolutiste et ses origines médiévales (bas MoyenAge)", 75-103, p. 131.

3. Thomas Smith, De Republica Anglorum (Londres, Henrie Midleton, 1583, The Scolar Press, 1970) 6, chap. 7: «A tyraunt they name him, who by force commeth to the Monarchy against the will of the people, breaketh lawes made at his pleasure, maketh other without the advise and consent of the people, and regardeth not the wealth of his communes but the advancement of himself, his faction, \& hindred».

4. R. Mousnier, L'Assassinat d'Henri IV (Paris, Gallimard, 1964); P. Ariès, Essais sur l'histoire de la mort en Occident du MoyenAge à nos jours (Paris, Seuil, 1975); M. Cohn, Europe's Inner Demons (Great-Britain, Sussex Univ. Press \& Heinemann Educational Books, 1975); C. Blum, "Recherches sur les fonctions épistémologiques d'une représentation allégorique : l'exemple de l'apparition en Occident de 
l'Allégorie de la Mort en squelette", Journal of Medieval and Renaissance Studies, 15 (1985), 13-27).

5. A. Boureau, Le simple corps du Roi. L'impossible sacralité des souverains français. XVe-XVIII siècles (Paris, Les Editions de Paris, 1988): "Le temps du corps propre, 1350-1550", 43-70, p. 59.

6. O. Ranum, "The French Ritual of Tyranicide in the Late Sixteenth Century", Sixteenth Century Journal, XI, $\mathrm{n}^{\circ} 1,1980$. [Exemple des corps des Guise, brûlés à la chaux vive puis jetés dans la Loire, de Ravaillac démembré, de façon à sentir son âme partir, de Concini, dont le cadavre fut retiré de la tombe pour être soumis à des génuflexions vexatoires et à une deuxième mort sous la forme d'une pendaison exemplaire.]

7. Les Essais de Montaigne, ed. P. Villey, reéde. par V.L. Saulnier (Paris, PUF, 1924, 1978), tome I, chap. XXXI, 209.

8. P. Camporesi, La Chair impassible, (Milan, il Saggiatore, 1983, Paris, Flammarion, Nouvelle Bibliothèque Scientifique, 1986); G. Mathieu-Castellani, Emblèmes de la Mort. Le dialogue de l'image et du texte (Paris, Nizet, 1988) chap. VII : "La Leçon d'anatomie", 123 138.

9. ROM, V. ii. 29. Les citations renvoient à l'édition Arden de chaque pièce. Le texte est celui de The First Folio of Shakespeare, The Norton Facsimile, ed. C. Hinman (Londres, N.Y., Sidney, Toronto, 1968). La proposition d'usage de cor(p)se(s) par rapport à body/bodies dans le théâtre de Shakespeare est de un à huit. Voir M. Spevack, The Harvard Concordance to Shakespeare (Belknap Press of Harvard Univ. Press, Cambridge, Mass., 1973).

10. Sur cette superstition, A. Boureau, Le simple corps du Roi, 69, n. 37, cite H. Platelle dans La Piété populaire. Voir R3, I ii 5567 : Le corps d'Henri VI saigne à la vue de son bourreau; ROM, III. ii. 54, 128 (le "corse" de Tybalt); IV-V 80, 89, 93; V. ii. 29 (le "corse" de Juliette).

11. $1 \mathrm{H} 4$, V. iv. 88 (le corps d'Hotspur); IV i 123 (le "corse" d'Hotspur); I. i. 43 (les "corse" des soldats de Mortimer).

12. C.G. Dubois, L'Imaginaire à la Renaissance (Paris, PUF, 1985), "Physis et Polis : les parents symboliques", 155-192.

13. Cor. II. i. 149; III. iii. 50; IV. v. 108 (le corps de Coriolan).

14. Cor. I. i. $95,104,121,133$; II. ii. 53; II. iii. 179; III. i. 153 (la cité, corps politique).

15. A. Baudart, "La Démocratie, tyrannie de l'insensé : une lecture de Platon", La Tyrannie, 23-38.

16. Cor. I. i. 89; I. i. 93 (la fable de Menenius est reprise ironiquement par le premier citoyen).

17. C.G. Dubois, L'Imaginaire, 175.

18. Cor. V. vi. 113 . 
19. L. Marin, Le Portrait du Roi (Paris, Editions de Minuit, 1987); O. Reboul, "Rhétorique et Pouvoir", La Tyrannie, 255.

20. Cor. V. vi. 8.

21. Cor. III. iii. 1-5; V. iii. 40-43.

22. Cor. II. iii. 179; Cor. V. vi. 57-59.

23. E.H. Kantorowicz, Les Deux Corps du Roi, trad. française par J.P. et N. Genet (Princeton Univ. Press, 1957, Paris, Gallimard, 1989), VII. "Le Roi ne meurt jamais", 228-325; R.E. Giesey, Le Roi ne meurt jamais, trad. française de D. Ebdnöther (The Royal Funeral Ceremony in Renaissance France, Travaux d'Humanisme et Renaissance, Genève, 1960, Paris, Flammarion 1987), chap. VI : "L'adoption de l'effigie funèbre", 127-163.

24. Cor. V. vi. 141-144.

25. E.H. Kantorowicz, Les Deux Corps, 300-301.

26. R.E. Giesey, Cérémonial et puissance souveraine. France $X V^{e}$-XVIIe siècles (Cahiers des Annales, Paris, A. Colin, 1987).

27. Ham., V. ii. $360-363,400-405$ (cp. Cor., V. vi. 147-150).

28. R. Gardette, "Shakespeare et la mort de César", R. Chevallier ed., Présence de César (coll. Caesarodunum XXbis, Paris, Les Belles Lettres, 1985) 119-136́.

29. A. Lascombes, "Obscur et glorieux": le corps théâtralisé dans Henry $I V$ Première partie", Henry the Fourth, Milton, Gay. Pouvoir et Musique (Cahiers du Gretes Univ. de Lille III Charles de Gaulle, 1990) 175-190: "synergie de l'opsis et de la mise en discours", 176.

30. J.C., I. ii. 210; I. ii. 133-139; I. ii. 118-127.

31. A. Lascombes, "Obscur ou glorieux", 177, 183 et 190, n. 6; l'auteur fait allusion à : Ezra Pound, A.B.C. of Reading (London, Faber and Faber, 1951), 42: «phanopoeia [throwing a visual image on the mind]». Voir aussi : M. Donker \& F.M. Muldrow, Dictionary of Literary \& Rhetorical Conventions of the English Renaissance (Wesport Connecticut, London Greenwood Press 1982) : "Ut Pictura Poesis", 238-241 [définitions de l'Enargia].

32. L. Strauss, De la tyrannie, trad. française de H. Ker (On Tyranny : An Interpretation of Xenophon's Hieron, New York Political Science Classics, 1948, Paris, Gallimard, 1954). C. Mossé, La Tyrannie dans la Grèce antique (Paris, PUF, 1969); M.F. Balez, "Mythe du tyran et réalités de l'Etat tyrannique dans l'Antiquité grecque", Tyrannie, despotisme, dictature (Inst. de Recherches sur les Civilisations de l'Occident moderne, Univ. de Paris-Sorbonne, 1984).

33. J.C., I. iii. 89-100; IV. iii. 99-106.

34. J. Auffret: "The Philosophic Background of Julius Caesar (Cahiers Elisabéthains $\left.n^{\circ} 5,1974\right) 73-74$. L'auteur note le parallélisme entre l'apologie du suicide par Cassius et dans Sénèque: Consolation à Marcia, 20, 2-3. 
35. J.C., II. i. 169-170; V. iii. 94-96.

36. J. Auffret, "The Philosophical Background of J.C.", 84.

37. J.C., II. ii. 13-26; V. i. 77-89.

38. J.C., I. ii. 116; V. iii. 91 \& 104 (le corps de Cassius); III. i. 228, 244, 253; III. ii. 42, 167, 256, 258; V. iii. 10 (le corps de César).

39. J.C., III. i. 199, 291.

40. E.H. Kantorowicz, Les Deux Corps, 247.

41. J.C., III. ii. 59-61.

42 J.C., III. ii. 77-78: «The evill that men do, lives after them, / The good is oft enterred with their bones».

43. J.C., III. ii. $160-161$.

44. E.H. Kantorowicz, Les Deux Corps, 269. Tyran]

45. Platon, La République, IX 577, c-e [L'âme tyrannisée du

46. J. Auffret, "The Philosophical Background of J.C.", 83-84.

47. E.R. Harvey, The Inward Wits. Psychological Theory in the Middle Ages and the Renaissance (London, The Warburg Institute, Warburg Inst. Essays, VI, 1975).

48. R.H. West, The Invisible Worlds. A Study of Pneumatology in Elizabethan Drama (The Univ. of Georgia Press, Athens 1939, The Folcroft Press, 1970).

49. R. Klibansky, E. Panofsky, F. Saxl, Saturne et la Mélancolie, trad. française de F. Durand-Bogaert et L. Evrard (Saturn and Melancholy. Studies of Natural Philosophy, Religion and Art, Th. Nelson \& Sons, 1964, Klaus Reprints, 1979, Paris, Gallimard, Bibliothèque Illustrée des Histoires, 1989).

50. Ham., I. ii. 87-107.

51. Ham., I. ii. 105.

52. Ham., I. iv. 51-57.

53. Ham., III. iv. 113-114: «O step between her, and her fighting Soule, / Conseit in weakest bodies, strongest workes».

54. Ham., III. iv. 18; III. iv. 139-141: "This is the very coynage of your Braine, / This bodiless Creation extasie is very cunning in»: «Monnaie que frappe ton cerveau. / Ces créatures sans corps / Le délire excelle à les forger.» Hamlet, texte français de J.M. Déprats, (Granit, Paris, 1986), p. 131.

55. Ham., III. iv. 54 : «The counterfet presentment of two Brothers".

56. Ham., III. ii. 24 (le corps du Temps); Ham., III. iv. 46 (le corps d'un contrat de mariage).

57. Ham., V. i. 166.

58. Ham., IV. i. 24, 36; IV. ii. 24, 26, 27; IV. iii. 1, 12 (le corps de Polonius); V. ii. 46-47, 376 (le corps absent de Rosencrantz et Guildenstern); V. ii. 330-332 (le corps de Claudius, contraint à l'union 
avec Certrude dans la mort); I. iv. 82 (le corps d'Hamlet, appelé à suivre le fantôme); I. v. 67, 73 (le corps du roi Hamlet détruit par le poison); V. ii. 382, 406 (les corps des victimes de la tragédie de la vengeance, dont Hamlet).

59. P. Camporesi, L'Officine des sens. Une anthologie baroque, trad. française de M. Bouhazer (Garzant Editore, s.p.a.s 1985, Paris, Hachette, 1989), p. 39.

60. Hal., V. i. $160,166,213$.

61. Voir par exemple: Art et Science de bien vivre et bien mourir (Rouen, Chez Jean Creuel, 1580): «des quinze Signes precedens le Jugement general et des joyes de Paradis», 125 , sig. $\mathrm{Zi}$; V. Marle, Iconographie de l'art profane du Moyen-Age et à la Renaissance, et la décoration des demeures (La Haye, 1931), vol. II. Iconographie : Rencontre des morts et des vifs 384-391, "la danse macabre des morts" (gravures sur bois de Wolgemut) 375, fig. 408.

62. Ham., II. ii. 263-264.

63. Etienne Junius Brutus [Hubert Languet], Vindiciae contra tyrannos, trad. française de 1581, ed. A. Jouanna, J. Perrin, M. Soulié, A. Tournon et H. Weber, coordinateur (Genève, Droz, 1979) 33.

64. Buchanan, De jure regni apud Scotos. Or a Dialogue Concerning the Due Privilege of Government in the Kingdom of Scotland (1579, ouvrage interdit en 1584), trad. anglaise de Philalethes (Londres, Richar Baldwin, 1689) 9. «For the Government of Kings is according to Nature, but that of Tyrants is not. A King doth rule his Subjects, and reign over them by their own consent. Tyrants reign over them, nill they will they. A Kingdom is a principality of a Free Man among Free Men : Tyranny is a principality of a Master over his slaves.»

65. C.H. Mac llwain, ed., The Political Works of James I (Harward Political Classics, I, 1918) 495 : «So if we take the whole people as one body and Masse, then as the Head is ordeined for the body and not the Body for the Head, so must a righteous King know himselfe to be ordained for his people and not his people for him» [A. Speach ... Delivered in the Upper House of the Parliament ... XIX March 1603].

66. M. Bloch, Les Rois Thaumaturges. Etudes sur le caractère surnaturel attribué à la puissance royale, particulièrement en France et en Angleterre (Publication de la Faculté des Lettres de Strasbourg, 1924, Paris, A. Colin, 1981, Gallimard, Bibliothèque des Histoires, 1988) 336-339. L'auteur rappelle que dans Macbeth, parmi les huit apparitions prophétiques, la dernière, celle de Jacques, fait l'objet d'une allusion au pouvoir thaumaturgique, Mac., IV.ii.139-159.

67. Par exemple John Po(y)net, A Shorte Treatise of Politike Power (Strasbourg, 1556, London, the Scolar Press, 1970). Sig. A6r : «And the wonderfull providence of God is herein to be wel noted and 
considered, of all suche as love and feare God, that in all places and countreyes wher God worde hathe ben receaved and embraced, ther for the tyme the people folowed God, no tirannye could entre, but all the members of the body fought the prosperitie and wealthe one of an other, for Goddes worde taught them so to doom.

68. King Richard III, ed. A. Hammond, The Arden Edition (London \& N.Y., Methuen, 1981) 54-61; King Richard II, ed. A. Gurr, The New Cambridge (Cambridge Univ. Press, 1984) 1-4.

69. F. Laroque, "La Notion de "misrule“ à l'époque élisabéthaine : la fête comme monde à l'envers et comme contre-temps", L'Image du monde renversé et ses représentations littéraires et paralittéraires de la fin du XVIe siècle au milieu du XVIIe, ed. J. Lafond et A. Redondo (Tours 1977, De Pétrarque à Descartes, Paris, Vrin, 1979) 161-170.

70. R.3, I.ii.32-33, 36-37: «Villaines set downe the Coarse, or by S. Paul, / Ile make a Coarse of him that disobeyes»; 229 (le corpseffigie d'Henri VI, plus tard remémoré par Anne, IV.i.66). La scène rappelle le rite funéraire écourté, où la dépouille d'Henri $\mathrm{V}$ est le témoin des déchirements de son royaume, H.6, I.i.62-64.

71. R.3, I.ii.46-48.

72. R.3, II.i.80-81.

73. R.3, V.iii.266-267.

74. R.3, V.iii.119-177.

75. G. Agamben, Stanze. Parole et fantasme dans la culture occidentale (Torino, Giulio Einaudi, 1977, Paris, Christian Bourgois, 1981).

76. R.3, V.iii.231-232.

77. R.3, I.ii.262; III.vii.75; III.iv.62, 68-72 (le corps de Richard III).

78. R.3, I.iv.270 (le corps de Clarence); V.i.47; V.iii.127 (le corps d'Henri); V.i.12 (le corps de Buckingham) pour qui le jour du Jugement est le jour des morts); V.v.15 (le corps des nobles); IV.iv.57 (le corps de la Duchesse d'York); IV.iv.277 (les corps des jeunes princes assassinés). 